\title{
To compare different methods of preparation of platelet rich plasma (PRP) and to analyse the correlation between initial $C B C$ and final yield of PRP
}

\section{Background}

Platelet rich plasma is an autologous preparation that concentrates the platelets in small volume. ${ }^{1}$ There are various studies, which have demonstrated platelet rich plasma to be a useful adjuvant in the treatment of disorders in dermatology, dentistry, orthopedic and sports medicine, oral and maxillary surgery and cardiac surgery. ${ }^{2-6}$ Though there are no controversies regarding the efficacy of PRP, there is dearth of studies which describe standardized techniques or methods to obtain PRP. Since PRP is obtained by centrifugation of the whole blood, there could be a possible correlation between initial platelet count, hematocrit and ESR in harvesting platelet rich plasma.

\section{Aims and objectives}

1. To compare three different methods of preparation of PRP and find out which method yields highest concentration of platelets.

2. To analyze if there is any correlation between platelet count, WBC count \& ESR and the method of preparation of platelet rich plasma employed.

\section{Materials and method}

It was a pilot analysis. 30 healthy volunteers were taken as study population.

\section{Inclusion criteria}

a) Healthy individuals in the age group of 18-40 years.

b) Individual in whom blood counts (WBC, RBC, platelets, ESR) are within normal limit.

c) Who are willing to participate in the study.

\section{Exclusion criteria}

a) High WBC count.

b) Any illness in the past 2 months

c) Intake of any antiplatelet drugs

d) Immunocompromised

e) Pregnancy and lactation

f) Donated blood in last 3 months

Methodology: 20ml of blood was drawn from each healthy volunteer after taking an informed written consent. The blood from each volunteer was collected in 4 separate sodium citrate (anticoagulant) containing test tubes. One test tube was used for the evaluation of complete blood count and blood from other three test tubes was subjected to three different methods of platelet rich plasma preparation as per the following guidelines:
Volume 3 Issue $3-2019$

\author{
Shilpa Dastikop, Santoshi MN, Sushruth \\ Kamoji \\ Department of Dermatology, Belagavi Institute of Medical \\ Sciences, India
}

\section{Correspondence: Sushruth Kamoji, Department of Dermatology, Belagavi Institute of Medical Sciences, India,} Email drsushruthk@yahoo.co.in

Received: May 31, 2019 | Published: June 20, 2019

Method 1: Double spin method; as suggested by Amanda et al., ${ }^{8}$ $1 \mathrm{st}$ spin at $100 \mathrm{~g}$ for $10 \mathrm{~min}$

$2 \mathrm{nd}$ spin at $400 \mathrm{~g}$ for $10 \mathrm{~min}$

Method 2: Double spin method; as suggested by Jun Arki et al.,9

1 st spin at $230-270 \mathrm{~g}$ for $10 \mathrm{~min}$

$2 \mathrm{nd}$ spin at $2300 \mathrm{~g}$ for $10 \mathrm{~min}$

Method 3: Single spin method; as suggested by Anitua et al., ${ }^{10}$

At $480 \mathrm{~g}$ for $10 \mathrm{~min}$

The conversion factor from ' $\mathrm{g}$ ' to rpm is as follows

$\mathrm{g}=(1.18) \times\left(10^{-5}\right) \mathrm{X}\left(\mathrm{RS}^{2}\right)$

Where, R- diameter of the rotation and S-speed in rpm(rotations per minute)

We used REMI-8C centrifuge machine for this study.

The process of centrifugation of all 3 methods was carried out in an air-conditioned environment at 24 degree Celsius. The platelet rich plasma which was obtained with each of the above method was subjected to analysis for platelet count, WBC and RBC count. Initial parameters of whole blood were compared with that method of PRP which yielded the highest concentration of platelets to check if there is any possible association between them.

\section{Results}

\section{Statistical analysis}

Data was entered into microsoft excel data sheet and was analyzed using SPSS 2 version software. Categorical data was represented in the form of frequencies and proportions. Chi-square was used as test of significance. Continuous data was represented as mean and standard deviation. Paired $t$ test is the test of significance for paired 
data. Pearson correlation was used to find the correlation between two quantitative variables. $\mathrm{P}$ value $<0.05$ was considered as statistically significant. Patients were in the age group of $18-40$ years. Mean age was 24.2 years (Table 1-4)(Figures1-3).

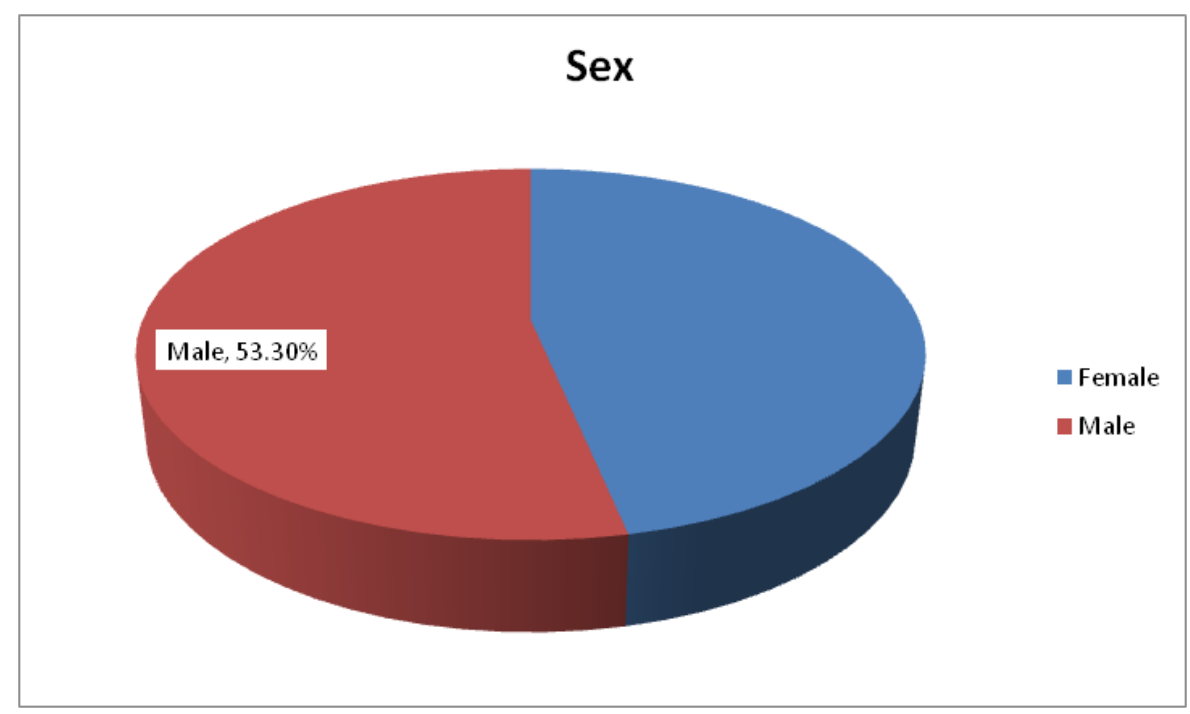

Figure I Pie chart depicting sex distribution of subjects in the study.

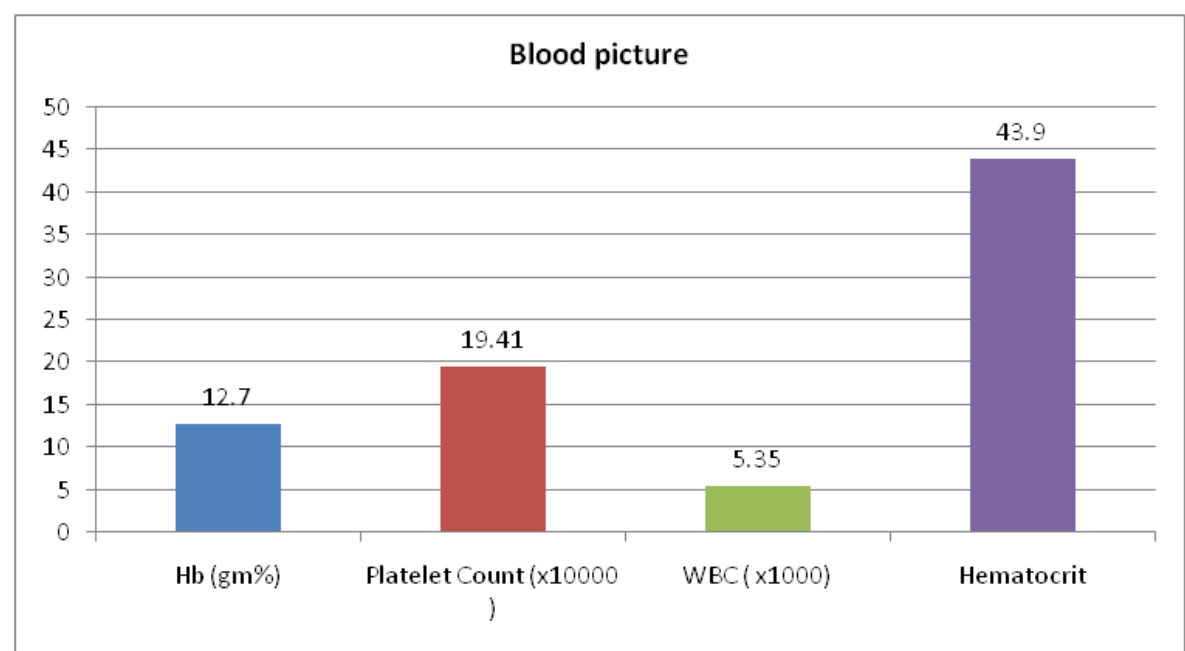

Figure 2 Bar graph showing blood picture among subjects.

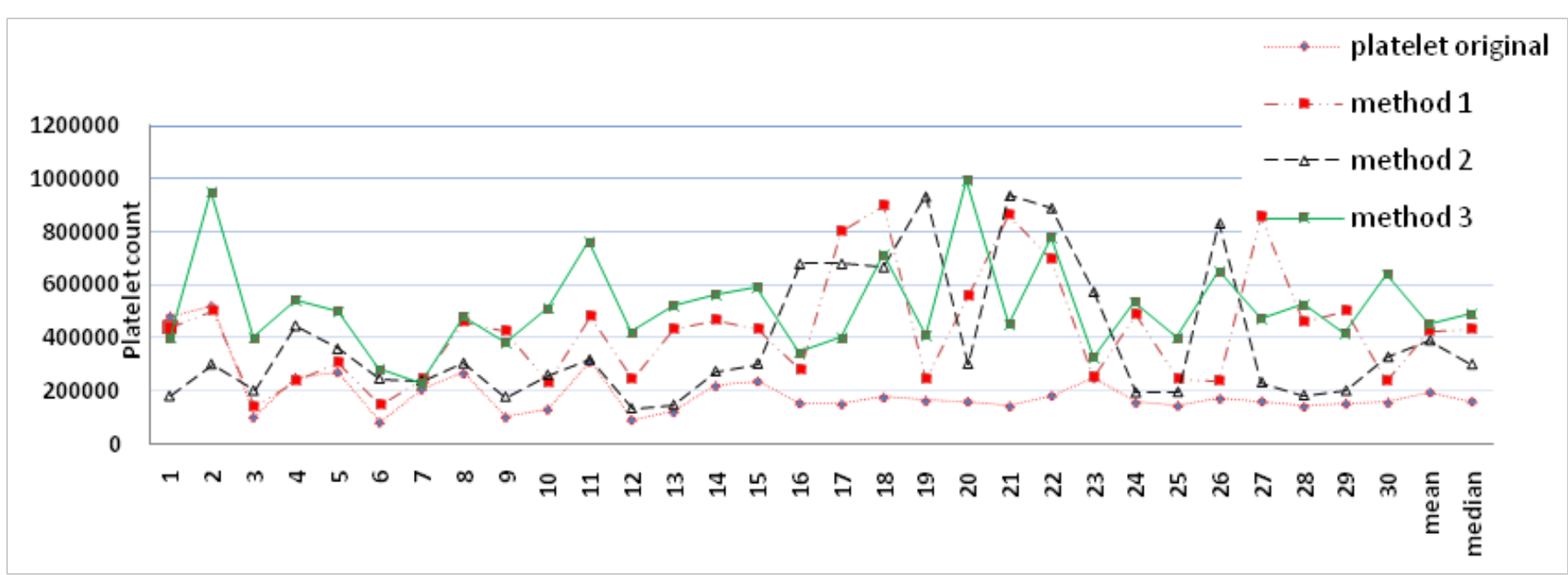

Figure 3 Graph showing Platelet yield with different methods plotted against baseline platelet count. 
Table I shows the age distribution of subjects in the study

\begin{tabular}{lllll}
\hline & Mean & SD & Median & Range \\
\hline Age(years) & 24.2 & 4.7 & 23.0 & 21.0
\end{tabular}

Table 2 Platelet concentrates by different methods of PRP extraction. It is evident from table 2 that platelet concentrate yield was maximum with method 3

\begin{tabular}{lllll}
\hline & Mean & SD & Median & Range \\
\hline Double spin method $(400 \mathrm{~g})$ & 428533.3 & 216618.5 & 434000 & 766000 \\
Double spin method $(2300 \mathrm{~g})$ & 390433.3 & 254441.9 & 300500 & 803000 \\
Single spin method & 518700 & 179855.1 & 489500 & 766000 \\
\hline
\end{tabular}

It is evident that platelet concentrate yield was maximum with single spin method.

Table 3 Comparison of platelet concentrates in different methods. Significant difference in mean platelet concentrate was observed between method 2 vs method 3 and between method I vs method 3. Overall method 3 had highest concentrate and method 2 had lowest concentrate.

\begin{tabular}{|c|c|c|c|c|c|}
\hline & Mean & $\mathbf{N}$ & Sd & Std. Error mean & $P$ value \\
\hline Double spin (method I) & 428533.33 & 30 & 216618.484 & 39548.943 & \multirow[b]{2}{*}{0.467} \\
\hline $\begin{array}{l}\text { vs } \\
\text { Double spin( method 2) }\end{array}$ & 390433.33 & 30 & 254441.875 & 46454.518 & \\
\hline Double spin(method 2) & 390433.33 & 30 & $25444 I .875$ & 46454.518 & \multirow[b]{2}{*}{$0.025^{*}$} \\
\hline $\begin{array}{l}\text { vs } \\
\text { Single spin( method 3) }\end{array}$ & 518700.00 & 30 & I79855.| 143 & 32836.906 & \\
\hline Double spin(method I) & 428533.33 & 30 & 216618.484 & 39548.943 & \multirow[b]{2}{*}{$0.039 *$} \\
\hline $\begin{array}{l}\text { vs } \\
\text { Single spin(method 3) }\end{array}$ & 518700.00 & 30 & I79855.143 & 32836.906 & \\
\hline
\end{tabular}

Table 4 Correlation between platelet concentrates by different methods with platelet count,WBC and hematocrit. From the above table it is clear that there was no significant correlation between platelet concentrate and $\mathrm{Hb} \%$ / initial platelet count/ WBC/hematocrit. However, only in method 2 there was decrease in platelet concentrate with increasing $\mathrm{Hb} \%$.

\begin{tabular}{|c|c|c|c|c|c|}
\hline & & Final Platelet count & Baseline $\mathbf{H b}$ & Baseline WBC & Baseline Hematocrit \\
\hline \multirow{3}{*}{ Double spin method I } & Pearson correlation & 0.079 & 0.022 & -0.076 & 0.154 \\
\hline & $P$ value & 0.677 & 0.909 & 0.691 & 0.417 \\
\hline & $\mathrm{N}$ & 30 & 30 & 30 & 30 \\
\hline \multirow{3}{*}{ Double spin method 2} & Pearson correlation & -0.057 & $-0.37 \mid *$ & 0.013 & 0.019 \\
\hline & $P$ value & 0.767 & $0.043^{*}$ & 0.945 & 0.921 \\
\hline & $\mathrm{N}$ & 30 & 30 & 30 & 30 \\
\hline \multirow{3}{*}{ Single spin method } & Pearson correlation & 0.348 & 0.127 & 0.338 & -0.008 \\
\hline & $P$ value & 0.059 & 0.505 & 0.068 & 0.967 \\
\hline & $\mathrm{N}$ & 30 & 30 & 30 & 30 \\
\hline
\end{tabular}

\section{Discussion}

Platelet rich plasma or platelet concentrates are used as adjuvant therapy in various fields of medicine including dermatology. These are supported by a number of studies which establish the role of PRP in various indications. However, there is paucity of studies to clearly define the method of preparing platelet rich plasma. In this study we compared 3 most widely quoted studies of preparation of platelet rich plasma to evaluate which one of these gave the highest yield of platelets. The results were contradictory. The single spin method gave the highest yield of platelets. The platelet concentration attained was consistent with the study done by Anuita et al., ${ }^{10}$ but contrary to most other studies. ${ }^{8,9}$
We also tried to find out if there was any correlation between the blood parameters of an individual and the platelet concentration obtained by different method. But no such statistically significant association was found. Using the centrifugation process to separate the blood component is The principles based on the differences in specific gravity of platelets and RBCs. This difference in specific gravity can be used for a differential sedimentation by varying the speed of rotation (RPM) and the duration of spin. This forms the basis of the facts that platelet concentration can be changed by changing the centrifugation force applied. Accordingly, a double spin method was described. The first centrifugation was aimed to separate RBC from other segments of the whole blood. Since RBCs are red, visible, and heavy; low speed (RPM) and short time would separate RBCs easily. 
230-270 $g$ for 10 minutes is reported to be the most efficient for first centrifugation. The second spin of higher RPM and duration would precipitate the platelets leading to platelet rich plasma. However, there is a risk of activation of platelets if the spin is too high.

Anitua et al. used only one centrifugation spin step and obtained a platelet concentration factor of 2.67. This was consistent with results obtained in our study.

Jo et al achieved a platelet concentration of $310.7 \pm 78.5 \times 10^{3} / \mathrm{mm}^{3}$ by using $900 \mathrm{~g}$ for $5 \mathrm{~min}$ for the first spin and of $1500 \times \mathrm{g}$ for $15 \mathrm{~min}$ second spin. The concentration achieved was 4.2 times greater than the baseline concentration. But in our study this method did not yield such high concentration. The maximum concentration achieved was almost 990000 and mean concentration 518700. It was much lower than previous reports.

Results of Amanda et al. showed that it is possible to achieve a concentration factor that is 5 times greater than baseline by applying a lower centrifugal acceleration in the second spin step $(400 \times \mathrm{g})$ for less time (10min) and concluded that efficient conditions for platelet recovery are low centrifugal acceleration (close to $100 \times \mathrm{g}, 10$ minutes) in the first spin and around $400 \times \mathrm{g}$ in the second spin for preventing effects on activating platelets. But in our study the concentration factor was 1.3 for the first method and 1.5 for the second method. Our study showed single spin method was superior in attaining higher concentrations compared to two other double spin methods. The single spin method also has few more added advantages. They are as follows:

1. As there is no need of transferring the solution from one test tube to another and once the blood is withdrawn from the patient, there is no handling of the sample either by the needle or by the pipette, asepsis required for the reinjection will be maintained.

2. Reduces the time required to obtain the PRP from almost $25 \mathrm{~min}$ to $10 \mathrm{~min}$.

3. Possibly reduces the decay of growth factors

4. Platelet yield at par with double spin

5. Volume of blood required is less compared to double spin method

6. More convenient to the treating dermatologist as well as the patient

7. Making it easier to prepare, which is helpful in providing this therapy in outpatient clinic.

8. It is more convenient to the treating dermatologist and is more safer than double spin method

\section{Limitations of the study}

It was a pilot analysis, large scale studies are required in order to standardize the procedure of PRP preparation.
Many of the previously done studies involving in vivo use of PRP preparation have used ACD-A as anticoagulant but in our study we have used sodium citrate for the same. Since this study was conducted in a resource poor setting we were unable to determine the platelet recovery rate.

\section{Acknowledgments}

None.

\section{Conflicts of interest}

Author declares that there is no conflict of interest.

\section{References}

1. Engebretsen L, Steffen K, Alsousou J, et al. IOC consensus paper on the use of platelet-rich plasma in sports medicine. Br J Sports Med. 2010;44(15):1072-1081.

2. Anitua E. Plasma rich in growth factors: preliminary results of use in the preparation of future sites for implants. Int J Oral Maxillofac Implants. 1999;14(4):529-535.

3. Gentile P, Bottini DJ, Spallone D, et al. Application of platelet-rich plasma in maxillofacial surgery:clinical evaluation. $J$ Craniofac Surg. 2010;21(3):900-904

4. Cervelli V, Gentile P, Scioli MG, et al. Application of platelet rich plasma in plastic surgery: clinical and in vitro evaluation. Tissue Eng C: Methods. 2009;15(4):625-634.

5. Dallari D, Savarino L, Stagni C, et al. Enhanced tibial osteotomy healing with use of bone grafts supplemented with platelet gel or platelet gel and bone marrow stromal cells. J Bone Joint Surg Am. 2007;89(11):24132420 .

6. Filardo G, Kon E, Ruiz MTP, et al. Platelet-rich plasma intra-articular injections for cartilage degeneration and osteoarthritis: single- versus double-spinning approach. Knee Surg Sports Traumatol Arthrosc. 2012;20(10):2082-2091.

7. Cieslik-Bielecka A, Bielecki T, Gazdzik TS, et al. Autologous platelets and leukocytes can improve healing of infected high-energy soft tissue injury. Transfus Apher Sci. 2009;41(1):9-12.

8. Perez AG, Lana JF, Rodrigues AA, et al. Relevant Aspects of Centrifugation Step in the Preparation of Platelet-Rich Plasma. ISRN Hematol. 2014;2014:176060.

9. Araki J, Jona M, Eto H, et al. Optimized Preparation Method of PlateletConcentrated Plasma and Noncoagulating Platelet-Derived Factor Concentrates: Maximization of Platelet Concentration and Removal of Fibrinogen. Tissue Eng: Part C methods. 2012;18(3):176-185.

10. Anitua E, Aguirre JJ, Algorta J, et al. Effectiveness of autologous preparation rich in growth factors for the treatment of chronic cutaneous ulcers. J Biomed Mater Res B: Appl Biomater. 2008;84(2):415-421. 